\title{
Resistance Training and Handball Players' Isokinetic, Isometric and Maximal Strength, Muscle Power and Throwing Ball Velocity: A Systematic Review and Meta-Analysis
}

\author{
Nicola Luigi Bragazzi $1, *,+\left(\mathbb{0}\right.$, Mehdi Rouissi ${ }^{2,+}$, Souhail Hermassi ${ }^{3, *, \ddagger}$ and \\ Karim Chamari $4,5, \ddagger(D)$ \\ 1 Department of Mathematics and Statistics, Laboratory for Industrial and Applied Mathematics (LIAM), \\ York University, Toronto, ON M3J 1P3, Canada \\ 2 Tunisian Research Laboratory, National Centre of Medicine and Science in Sport, Tunis 1003, Tunisia; \\ mehdirwissi@gmail.com \\ 3 Sport Science Program, College of Arts and Sciences, Qatar University, Doha 2713, Qatar \\ 4 ASPETAR, Orthopedic and Sports Medicine Hospital, Doha 29222, Qatar; karim.chamari@aspetar.com \\ 5 Laboratory "Sport Performance Optimization", National Center of Sports Medicine and Sports \\ Sciences (CNMSS), ISSEP Ksar-Said, Manouba University, Tunis 2010, Tunisia \\ * Correspondence: robertobragazzi@gmail.com (N.L.B.); shermassi@qu.edu.qa (S.H.) \\ + The first two authors are co-first authors. \\ $\ddagger$ The last two authors are co-last authors.
}

Received: 19 March 2020; Accepted: 2 April 2020; Published: 13 April 2020

\begin{abstract}
Purpose: Handball (Team Handball) is an intermittent and strenuous contact sport, the successful performance of which depends on frequent body contacts, and the ability to make repeated explosive muscular contractions required for jumping, acceleration, sprinting, turning, changing pace, and throwing a ball. Many studies have investigated the effect of resistance training (RT) in handball players, however with conflicting results. Therefore, our objective was to investigate the impact of RT on maximal strength (isometric and isokinetic strength), the power of both lower and upper limbs, and throwing velocity, in handball players. Methods: A comprehensive literature search yielded a pool of 18 studies, which were retained in the systematic review and meta-analysis. Results: A total of 275 handball players were included. The overall effect size (ES) of RT was 0.996 ([95\% CI 0.827-1.165], $p=0.0000$ ). At the multivariate meta-regression, the effect of publication year was significant, as well as the effects of country, gender, and level. The impact of RT on isokinetic strength was not significant (ES 0.079 [95\%CI -0.060-0.219], $p=0.265$ ), whereas the impact of RT on throwing (ES 1.360 [95\%CI $0.992-1.728], p=0.000$ ) was significant, as well as the effects of RT on isometric strength (ES 0.398 [95\%CI 0.096-0.700], $p=0.010$ ), on maximal strength (ES 1.824 [95\%CI 1.305-2.343], $p=0.000$ ), and on power (ES 0.892 [95\%CI 0.656-1.128], $p=0.000$ ). Conclusions: RT has a significant impact in handball players. Handball coaches could design conditioning protocols and programs based on our results. However, due to a number of shortcomings, including the high, statistically significant heterogeneity among studies and the evidence of publication bias, further high-quality investigations are needed.
\end{abstract}

Keywords: strength training; peak power; team handball; systematic review; meta-analysis

\section{Introduction}

Team handball is a sport that requires physical efforts of high-intensity and of short duration with a strenuous contact and a particular ability to make and repeat explosive muscular contractions. During a handball match, more than 825 high intensity actions can be performed, requiring a high 
level of strength [1-4]. Maximal strength, power and throwing velocity are considered as major determinants of success in elite handball players. Hence, handball coaches should perform specific handball conditioning, including high intensity exercises such as resistance training (RT) to develop these physical qualities. RT involves the use of wide range of resistive loads and a variety of training modalities (i.e., throwing with regular balls, throwing with a medicine ball, Olympic weight lifting, elastic band training and plyometric training) aimed at developing maximal strength and/or muscular power.

Throwing is a fundamental skill in handball. Two basic factors influence the efficiency of shots: accuracy and throwing ball velocity. The faster the ball is thrown, the less time defenders and/or goal keeper have to save the shot. Coaches and scientists seem to have reached an agreement stating that the main determinants of throwing ball velocity are the timing of movement in consecutive body segments, the technique used, and the strength and power of both the upper and lower limbs [1-3]. Each of these factors can be improved by training, particularly RT designed to enhance strength and power in both the upper and lower limbs. However, training programs that produce the greatest change in muscle cross-section typically involve loads of $70 \%$ with one repetition maximum (1RM) [2], whereas programs designed to improve strength through enhanced neuronal coordination are characterized by intensities of $85-100 \%$ 1RM [2].

Many studies have investigated the effect of RT on throwing velocity in handball players, but with conflicting results. However, differences in the intensity of training may also have contributed to conflicting results. For instance, Chelly et al. [5] indicated that upper limb plyometric training improved throwing ball performance in the RT trained group, with no significant improvement in the control group, whereas, for instance, other studies noted a significant enhancement $(p<0.001)$ in standing handball throwing velocity after 6 weeks of heavy upper limb RT. Furthermore, Hermassi et al. [6-8] reported similar results after RT programs. On the other hand, Raeder et al. [9] reported a significant increase in throwing velocity of $14 \%$ and $3.7 \%$ for both training and control groups, respectively. Genevois et al. [10] indicated non-significant improvements in throwing ball velocity for the dominant arm following 6 weeks of a strengthening program.

Moreover, the major role of the lower limb strength and power has also been highlighted in many studies demonstrating a significant relationship between such high intensity actions and lower limb strength/power [11-13]. To maintain a high level of strength, both strength/power training should be carefully monitored throughout the competitive season. RT programs could be very useful to develop lower/upper limb strength and to maintain it throughout the season, both for male and female handball players [11-13]. Specific handball studies on this issue have examined the impact of speed strength programs on muscular power, jump performance or throwing velocity [5,11-15]. Gorostiaga et al. [15] showed that specific RT can improve the strength of both the upper extremity muscles $(23 \% ; p<0.01)$ and the leg extensors $(12.2 \% ; p<0.01)$, but no gains can be anticipated from the low resistance forms of activity involved in team handball practice. Marques and Gonzalez-Badillo [14] also noted a 28\% increase in 1RM bench press in high level handball players after 12 weeks of RT (2-3 sessions per week), close to the $34 \%$ gain in $1 \mathrm{RM}$ bench press reported by other studies [6]. Their loadings were in the range $70-85 \%$ of concentric $1 \mathrm{RM}$ bench press. Hermassi et al. [6] also found upper limb gains of $34 \%$ and $20 \%$ for pullover and bench press, respectively, over 8 weeks of strength training. This is a larger response than the results previously observed by Gorostiaga et al. [15].

In addition, Gorostiaga et al. [15] are the only previous investigators who have studied the influence of heavy RT on the jump performance of handball players. They reported significant increases in a group that had previously engaged only in team practice $(6 \% ; p<0.001)$, but no changes in counter movement jump (CMJ) for either RT or control groups. Hermassi et al. [6] observed a significant increase in the jump performance of well-trained handball athletes after 8 weeks of heavy squat training, associated with a large improvement in 1RM squat strength.

However, despite the popularity of handball and the importance of RT, no systematic review exists on the topic. Therefore, the objective of this systematic review and meta-analysis was to investigate the 
effects of RT on maximal strength (isometric and isokinetic strength), the power of both lower and upper limbs and throwing velocity in handball players, hopefully clarifying some of the discrepancies found in the literature. Thus, handball coaches could use this review as a guideline for designing specific RT programs aiming to improve or maintain force-related physical performance of handball players throughout the competitive season and to potentially also reduce the rate of injury.

\section{Materials and Methods}

A systematic review with meta-analysis was performed according to the "Preferred Reporting Items for Systematic reviews and Meta-Analyses" (PRISMA) guidelines [16]. The main review question was: what is the effect of RT on maximal strength, isometric and isokinetic strength, the power of lower and upper limbs and throwing velocity in handball players [17,18]?

In order to answer to this review question, a comprehensive search was carried out, systematically searching the following scholarly electronic databases: namely, PubMed/MEDLINE, the Cochrane Central Register of Controlled Trials (CENTRAL), Scopus and ISI/Web of Science. No limits were placed on publication date (in other words, no time filter was applied: all the scholarly databases were mined from inception). Articles written in any language were eligible. We also manually scanned the reference lists of articles identified by previous electronic searches and looked through the citation analysis in review papers regarding handball to increase the chance of including all relevant studies eventually missed by the original search terms.

The following Participants, Exposure, Comparator, Outcome and Study (PECOS) criteria were followed:

- $\quad$ Participants/population: we included studies where the study population consisted of handball athletes (either amateur or professional players), men or women, youths or adults;

- $\quad$ Exposure(s): we analyzed handball players exposed to RT;

- Comparator(s)/control: we analyzed other kinds of training versus RT;

- Outcome(s): the primary outcome was the effect of the RT on maximal strength, isometric or isokinetic strength, power and/or throwing velocity in handball players.

- Study design: we considered any study design except for studies designed as expert opinion, comment/commentary, editorial/letter to editor, and review.

Gray literature was not considered, since we preferred to focus on high-quality peer-reviewed investigations. Studies that duplicated populations from the same authors were also excluded, as well as studies with a lack of quantitative details or results that were not pertinent to the scope of the present review.

The primary search strategy included the following search terms: handball AND "resistance training" AND (maximal strength OR force OR power OR throwing velocity OR isometric strength OR isokinetic strength). Further details are shown in Table 1.

All data were independently extracted by two reviewers (M.R., N.L.B.) using an ad-hoc designed Excel spreadsheet and independently verified/confirmed by another reviewer, with expertise in the field of handball (K.C.). Firstly, screening of titles and/or abstracts for relevant and possibly eligible citations was performed. Secondly, full text article analysis was conducted to carefully select studies. In the case of disagreements, a consensus was reached through discussion. If disagreements were not resolved, a third reviewer (K.C.) was consulted and acted as final referee. 
Table 1. Search strategy adopted in the present systematic review and meta-analysis.

\begin{tabular}{|c|c|}
\hline Search Strategy Item & Details \\
\hline Searched keywords & $\begin{array}{l}\text { handball AND "resistance training" AND (maximal strength OR force OR } \\
\text { power OR throwing velocity OR isometric strength OR isokinetic strength) }\end{array}$ \\
\hline Searched databases & $\begin{array}{l}\text { PubMed/MEDLINE, the Cochrane Central Register of Controlled Trials } \\
\text { (CENTRAL), Scopus and ISI/Web of Science }\end{array}$ \\
\hline Inclusion criteria & $\begin{array}{l}\text { - } \quad \text { (Participants/population): handball athletes (either amateur or } \\
\text { professional players), men or women, youths or adults; } \\
\text { - } \quad \text { E (Exposures): handball players exposed to RT; } \\
\text { - } \quad \text { C (Comparator(s)/control): other kinds of training versus RT; } \\
\text { - } \quad \text { O (Outcomes): the effect of the RT on maximal strength, isometric or } \\
\text { isokinetic strength, power, throwing velocity; } \\
\text { - } \quad \text { S (Study design): Any experimental study design. }\end{array}$ \\
\hline Exclusion criteria & $\begin{array}{l}\text { - P (Participants/population): sports athletes other than handball players; } \\
\text { - } \quad \text { (Exposures): handball players exposed to training other than RT or } \\
\text { combined with RT, in which it was not possible to pull out the single } \\
\text { effects of RT; } \\
\text { - C (Comparator(s)/control): other kinds of training rather than RT } \\
\text { versus RT; } \\
\text { - O (Outcomes): the effect of the RT on other variables; } \\
\text { - S (Study design): expert opinion; comment/commentary; editorial/letter } \\
\text { to editor; review. }\end{array}$ \\
\hline
\end{tabular}

Abbreviation: resistance training (RT).

A meta-analysis has been performed, utilizing the commercial software "Comprehensive Meta-analysis" CMA version 3.0 (Biostat, Englewood, USA). The effect size (ES) was expressed as Hedges' g, setting a correlation between pre- and post-measures of 0.50, whereas the global ES was computed according to Morris. In cases of considerable heterogeneity $\left(\mathrm{I}^{2}>50 \%\right)$, a random effect model was carried out, instead of a fixed effect model. Evidence of publication bias was verified by both visually inspecting the funnel plot and computing Egger's linear regression test. The Duval and Tweedie trim-and-fill analysis was performed to compute the true ES, in cases where there was evidence of publication bias. Meta-regression analyses were conducted to shed light on the determinants of the ES.

Results are presented in a tabular form, together with an analysis of the different subgroups or subsets.

\section{Results}

\subsection{Systematic Review}

The initial search yielded 2570 studies. Finally, after deleting duplicates and based on inclusion/exclusion criteria, 18 studies [5-10,13-15,17,19-26] were retained in the present systematic review and meta-analysis (Figure 1). The main features of the studies are presented in Table 2. A total of 275 handball players were included. 
Table 2. Main characteristics of included studies.

\begin{tabular}{|c|c|c|c|c|c|c|c|c|c|c|c|c|c|c|}
\hline Authors & Year & Country & $\begin{array}{l}\text { Randomized } \\
\text { or Not } \\
\text { (R, NR) }\end{array}$ & Sample & $\begin{array}{c}\text { Drop-Out } \\
\text { Rate }\end{array}$ & Age & $\begin{array}{l}\text { Gender (in } \\
\text { Percentage) }\end{array}$ & $\begin{array}{l}\text { Anthropometric } \\
\text { Features of } \\
\text { Sample (Height, } \\
\text { Body Mass, } \\
\text { BMI, etc.) }\end{array}$ & Sport & $\begin{array}{c}\text { Level } \\
\text { (International, } \\
\text { National, } \\
\text { Elite) }\end{array}$ & $\begin{array}{l}\text { Type of } \\
\text { Training }\end{array}$ & $\begin{array}{l}\text { Duration of } \\
\text { Training }\end{array}$ & $\begin{array}{c}\text { Variable } \\
\text { Measured }\end{array}$ & $\begin{array}{l}\text { Statistical } \\
\text { Test Used }\end{array}$ \\
\hline $\begin{array}{c}\text { Gorostiaga } \\
\text { et al. [17] }\end{array}$ & 2005 & Spain & Randomized & 15 & 0 & $31 \pm 4$ & $100 \%$ men & $\begin{array}{c}\text { Body mass: } 95.6 \\
\pm 14.3 ; \text { Body fat } \\
(\%): 14.9 \pm 4.2 ; \\
\text { Height }(\mathrm{cm}): \\
188 \pm 7\end{array}$ & Handball & Elite & $\begin{array}{l}\text { Maximal } \\
\text { strength of the } \\
\text { upper extremity } \\
\text { was assessed } \\
\text { using one } \\
\text { repetition } \\
\text { concentric } \\
\text { maximal bench } \\
\text { press action }\end{array}$ & $\begin{array}{l}\text { 45-weeks in } \\
\text { season }\end{array}$ & $\begin{array}{l}\text { Vertical } \\
\text { jumping } \\
\text { height, } \\
\text { throwing } \\
\text { velocity, } \\
\text { muscle power } \\
\text { output; } \\
\text { maximal } \\
\text { strength of } \\
\text { upper and } \\
\text { lower limb }\end{array}$ & $\begin{array}{l}\text { ANOVA with } \\
\text { repeated } \\
\text { measures was } \\
\text { used to determine } \\
\text { the differences } \\
\text { between tests. } \\
\text { When a } \\
\text { significant F } \\
\text { value was } \\
\text { achieved, } \\
\text { appropriate } \\
\text { Scheffe's post-hoc } \\
\text { tests procedures } \\
\text { were used to } \\
\text { locate the } \\
\text { difference } \\
\text { between means }\end{array}$ \\
\hline $\begin{array}{l}\text { Holm } \\
\text { et al. [23] }\end{array}$ & 2004 & Norway & Randomized & 35 & 0 & $23 \pm 2.5$ & $\begin{array}{c}100 \% \\
\text { women }\end{array}$ & $\begin{array}{c}\text { Body mass was } \\
69.2 \pm 7.3 \mathrm{~kg}\end{array}$ & Handball & Elite division & $\begin{array}{l}\text { Neuromuscular } \\
\text { training }\end{array}$ & $\begin{array}{l}\text { Three times a } \\
\text { week over } 8 \\
\text { weeks }\end{array}$ & $\begin{array}{l}\text { Balance, } \\
\text { muscle } \\
\text { strength of } \\
\text { lower limb }\end{array}$ & $\begin{array}{l}\text { A } 3 \text { (time) } \times 2 \\
\text { (leg) analysis of } \\
\text { variance for } \\
\text { repeated } \\
\text { measures (with } \\
\text { the least } \\
\text { significant } \\
\text { difference post } \\
\text { hoc test) was } \\
\text { used to calculate } \\
\text { differences from } \\
\text { one test to the } \\
\text { next over the } \\
\text { study period }\end{array}$ \\
\hline $\begin{array}{l}\text { Oxyzoglou } \\
\text { et al. [22] }\end{array}$ & 2007 & Greece & Randomized & 51 & 0 & $13.7 \pm 1.5$ & $100 \%$ men & $\begin{array}{l}\text { Height }=168.06 \\
\pm 7.8 \mathrm{~cm} ; \text { weight } \\
=56.90 \pm 10.35 \mathrm{~kg}\end{array}$ & Handball & $\begin{array}{l}\text { Pre-adolescent } \\
\text { athletes }\end{array}$ & $\begin{array}{c}\text { Various } \\
\text { shooting } \\
\text { throws with } \\
\text { horizontal and } \\
\text { vertical jumps } \\
\text { of different } \\
\text { height }\end{array}$ & $\begin{array}{c}\text { Three } \\
\text { sessions/week } \\
60 \text { min } \\
\text { 6-month }\end{array}$ & $\begin{array}{l}\text { Long jump, } \\
\text { vertical jump, } \\
\text { throwing of } \\
\text { medicine ball, } \\
\text { strength of } \\
\text { right hand } \\
\text { grip, strength } \\
\text { of left hand } \\
\text { grip, hanging } \\
\text { from a } \\
\text { horizontal bar, } \\
\text { body sit- up }\end{array}$ & $\begin{array}{l}\text { An analysis of } \\
\text { covariance } \\
\text { (ANCOVA) was } \\
\text { performed to } \\
\text { examine the } \\
\text { differences } \\
\text { between groups } \\
\text { in post-training } \\
\text { values where the } \\
\text { pre-training } \\
\text { mean was used as } \\
\text { a covariate }\end{array}$ \\
\hline
\end{tabular}


Table 2. Cont

\begin{tabular}{|c|c|c|c|c|c|c|c|c|c|c|c|c|c|c|}
\hline Authors & Year & Country & $\begin{array}{c}\text { Randomized } \\
\text { or Not } \\
\text { (R, NR) }\end{array}$ & Sample & $\begin{array}{c}\text { Drop-Out } \\
\text { Rate }\end{array}$ & Age & $\begin{array}{l}\text { Gender (in } \\
\text { Percentage) }\end{array}$ & $\begin{array}{l}\text { Anthropometric } \\
\text { Features of } \\
\text { Sample (Height, } \\
\text { Body Mass, } \\
\text { BMI, etc.) }\end{array}$ & Sport & $\begin{array}{c}\text { Level } \\
\text { (International, } \\
\text { National, } \\
\text { Elite) }\end{array}$ & $\begin{array}{c}\text { Type of } \\
\text { Training }\end{array}$ & $\begin{array}{l}\text { Duration of } \\
\text { Training }\end{array}$ & $\begin{array}{l}\text { Variable } \\
\text { Measured }\end{array}$ & $\begin{array}{l}\text { Statistical } \\
\text { Test Used }\end{array}$ \\
\hline $\begin{array}{l}\text { Ettema } \\
\text { et al. [21] }\end{array}$ & 2008 & Norway & Randomized & 19 & 6 players & $18.1 \pm 2.1$ & $\begin{array}{c}100 \% \\
\text { women }\end{array}$ & $\begin{array}{c}\text { Body mass } 64.0 \pm \\
7 \mathrm{~kg} \text {, height } 1.67 \\
\pm 0.03 \mathrm{~m}\end{array}$ & Handball & Sub-elite & $\begin{array}{c}\text { Pulley device } \\
\text { mimicking } \\
\text { throwing } \\
\text { kinematics at } \\
85 \% \text { of } 1 \mathrm{RM}\end{array}$ & $\begin{array}{c}\text { Three } \\
\text { sessions per } \\
\text { week over } 8 \\
\text { weeks }\end{array}$ & $\begin{array}{l}\text { Throwing } \\
\text { velocity }\end{array}$ & $\begin{array}{l}\text { A two-way } \\
\text { ANOVA } \\
\text { for repeated } \\
\text { measures was } \\
\text { used }\end{array}$ \\
\hline $\begin{array}{l}\text { Gorostiaga } \\
\text { et al. [15] }\end{array}$ & 1999 & Spain & $\begin{array}{c}\text { Not } \\
\text { mentioned }\end{array}$ & 24 & NO & $15.1 \pm 0.7$ & $100 \%$ men & $\begin{array}{c}\text { Body mass } 62.4 \pm \\
7 \mathrm{~kg} \text {, height } 1.73 \\
\pm 0.05 \mathrm{~m}, \text { and } \\
\text { body fat } 11.3 \pm \\
3.1 \%)\end{array}$ & Handball & Sub-elite & $\begin{array}{c}\text { Heavy-resistance } \\
\text { weight lifting } \\
40 \% \text { to } 90 \% \text { RM: } \\
\text { (bench press, } \\
\text { half squat, knee } \\
\text { fexion curl, leg } \\
\text { press and } \\
\text { pec-deck) }\end{array}$ & $\begin{array}{l}\text { Two sessions } \\
\text { per week over } \\
6 \text { weeks }\end{array}$ & $\begin{array}{l}\text { Throwing ball } \\
\text { velocity, squat } \\
\text { jump, } \\
\text { countermovement } \\
\text { jump, 1RM leg } \\
\text { press, } 1 \text { RM } \\
\text { pec-deck }\end{array}$ & $\begin{array}{l}\text { One-way } \\
\text { ANOVA }\end{array}$ \\
\hline $\begin{array}{l}\text { Hermassi } \\
\text { et al. [6] }\end{array}$ & 2010 & Tunisia & Randomized & 26 & NO & $20 \pm 0.6$ & $100 \%$ men & $\begin{array}{c}\text { Body mass } 85.0 \\
\pm 13.2 \mathrm{~kg} \\
\text { height } 1.86 \pm 0.06 \\
\mathrm{~m}, \text { and body fat } \\
13.7 \pm 2.4 \%)\end{array}$ & Handball & Elite & $\begin{array}{c}\text { Heavy resistance } \\
\text { training group } \\
\text { (80\% to } 95 \% \text { of } \\
1 \mathrm{RM}) \text { and } \\
\text { moderate } \\
\text { resistance } \\
\text { training group } \\
\text { (55\% to } 75 \% \text { of } \\
1 \mathrm{RM})\end{array}$ & $\begin{array}{c}\text { Two sessions } \\
\text { per week over } \\
10 \text { weeks }\end{array}$ & $\begin{array}{c}\text { Upper limb } \\
\text { power, handball } \\
\text { throwing velocity, } \\
\text { 1RM bench press, } \\
\text { 1RM pullover }\end{array}$ & $\begin{array}{l}\text { A two-way } \\
\text { analysis of } \\
\text { variance } \\
\text { (ANOVA) with } \\
\text { repeated measure } \\
\text { (group X time) }\end{array}$ \\
\hline $\begin{array}{l}\text { Hermassi } \\
\text { et al. [7] }\end{array}$ & 2011 & Tunisia & Randomized & 24 & 0 & $24 \pm 0.7$ & $100 \%$ men & $\begin{array}{c}\text { Height } 1.83 \pm 0.08 \\
\mathrm{~m}, \text { body mass } 81 \\
\pm 12 \mathrm{~kg}, \text { body fat } \\
13.2 \pm 1.3 \%\end{array}$ & Handball & Elite & $\begin{array}{l}\text { Heavy resistance } \\
\text { training ( } 80 \% \text { to } \\
95 \% \text { of } 1 \mathrm{RM}) \\
\text { group vs. } \\
\text { control group }\end{array}$ & $\begin{array}{c}\text { Two sessions } \\
\text { per week over } \\
8 \text { weeks }\end{array}$ & $\begin{array}{c}\text { Peak power } \\
\text { (cycle ergometer), } \\
\text { squat jump, } \\
\text { countermovement } \\
\text { jump, sprint tests, } \\
\text { 1RM bench press, } \\
\text { 1RM pullover, } \\
\text { 1RM back half } \\
\text { squat test }\end{array}$ & $\begin{array}{l}\text { Training effects } \\
\text { were assessed by } \\
\text { a one-way } \\
\text { analysis } \\
\text { of variance with } \\
\text { repeated measure } \\
\text { (group X time). }\end{array}$ \\
\hline
\end{tabular}


Table 2. Cont

\begin{tabular}{|c|c|c|c|c|c|c|c|c|c|c|c|c|c|c|}
\hline Authors & Year & Country & $\begin{array}{c}\text { Randomized } \\
\text { or Not } \\
\text { (R, NR) }\end{array}$ & Sample & $\begin{array}{l}\text { Drop-Out } \\
\text { Rate }\end{array}$ & Age & $\begin{array}{l}\text { Gender (in } \\
\text { Percentage) }\end{array}$ & $\begin{array}{l}\text { Anthropometric } \\
\text { Features of } \\
\text { Sample (Height, } \\
\text { Body Mass, } \\
\text { BMI, etc.) }\end{array}$ & Sport & $\begin{array}{c}\text { Level } \\
\text { (International, } \\
\text { National, } \\
\text { Elite) }\end{array}$ & $\begin{array}{c}\text { Type of } \\
\text { Training }\end{array}$ & $\begin{array}{l}\text { Duration of } \\
\text { Training }\end{array}$ & $\begin{array}{c}\text { Variable } \\
\text { Measured }\end{array}$ & $\begin{array}{l}\text { Statistical } \\
\text { Test Used }\end{array}$ \\
\hline $\begin{array}{l}\text { Hermassi } \\
\text { et al. [8] }\end{array}$ & 2014 & Tunisia & Randomized & 24 & 0 & $20 \pm 0.3$ & $100 \%$ men & $\begin{array}{c}\text { Body mass: } 89.1 \\
\pm 2.1 \mathrm{~kg}, \text { height: } \\
1.88 \pm 0.07 \mathrm{~m} \\
\text { body fat: } 13.2 \pm \\
1.3 \%)\end{array}$ & Handball & Elite & $\begin{array}{c}\text { Plyometric } \\
\text { training }\end{array}$ & $\begin{array}{l}\text { Two sessions } \\
\text { per week over } \\
8 \text { weeks }\end{array}$ & $\begin{array}{l}\text { Leg power (cycle } \\
\text { ergometer), } \\
\text { squat jump, } \\
\text { countermovement } \\
\text { jump }\end{array}$ & $\begin{array}{l}\text { To compare the } \\
\text { effects of the } \\
\text { plyometric training, } \\
\text { a mixed } \\
\text { design } 2 \text { (test } \\
\text { occasion: pre-post: } \\
\text { repeated measures) } \\
\times 2 \text { (group: } \\
\text { plyometric and } \\
\text { control) } \\
\text { Analysis of } \\
\text { variance (ANOVA) } \\
\text { for each } \\
\text { performance test } \\
\text { variable was used. } \\
\text { In addition, a } \\
\text { one-way ANOVA } \\
\text { with repeated } \\
\text { measures was } \\
\text { conducted for each } \\
\text { group to show if } \\
\text { changes } \\
\text { from pre- to } \\
\text { post-test in each } \\
\text { group were } \\
\text { significant. }\end{array}$ \\
\hline $\begin{array}{l}\text { Hermassi } \\
\text { et al. [25] }\end{array}$ & 2015 & Tunisia & Randomized & 34 & 0 & $18 \pm 0.5$ & $100 \%$ men & $\begin{array}{c}\text { Body mass: } 80.6 \\
\pm 5.5 \mathrm{~kg} \text {, height: } \\
1.80 \pm 5.1 \mathrm{~cm} \\
\text { body fat: } 13.4 \pm \\
0.6 \%)\end{array}$ & Handball & Elite & $\begin{array}{c}\text { Group 1: } \\
\text { throwing with } \\
\text { medicine ball of } \\
3 \mathrm{~kg} \text {, Group 2: } \\
\text { throwing with } \\
\text { regular ball, } \\
\text { control group }\end{array}$ & $\begin{array}{l}\text { Three } \\
\text { sessions per } \\
\text { week over } 8 \\
\text { weeks }\end{array}$ & $\begin{array}{l}\text { 1RM bench } \\
\text { press, 1RM pull } \\
\text { over+ ball } \\
\text { throwing } \\
\text { velocity and ball } \\
\text { throwing } \\
\text { distance }\end{array}$ & $\begin{array}{l}\text { To compare the } \\
\text { effects of the } \\
\text { training protocols, } \\
\text { a mixed-design 2 } \\
\text { (test occasion: } \\
\text { pre-post: } \\
\text { repeated measures) } \\
3 \text { (group: control, } \\
\text { regular throwing, } \\
\text { and resistance } \\
\text { training) ANOVA } \\
\text { on each variable } \\
\text { was used }\end{array}$ \\
\hline
\end{tabular}


Table 2. Cont

\begin{tabular}{|c|c|c|c|c|c|c|c|c|c|c|c|c|c|c|}
\hline Authors & Year & Country & $\begin{array}{l}\text { Randomized } \\
\text { or Not } \\
\text { (R, NR) }\end{array}$ & Sample & $\begin{array}{c}\text { Drop-Out } \\
\text { Rate }\end{array}$ & Age & $\begin{array}{l}\text { Gender (in } \\
\text { Percentage) }\end{array}$ & $\begin{array}{l}\text { Anthropometric } \\
\text { Features of } \\
\text { Sample (Height, } \\
\text { Body Mass, } \\
\text { BMI, etc.) }\end{array}$ & Sport & $\begin{array}{c}\text { Level } \\
\text { (International, } \\
\text { National, } \\
\text { Elite) }\end{array}$ & $\begin{array}{c}\text { Type of } \\
\text { Training }\end{array}$ & $\begin{array}{l}\text { Duration of } \\
\text { Training }\end{array}$ & $\begin{array}{l}\text { Variable } \\
\text { Measured }\end{array}$ & $\begin{array}{l}\text { Statistical } \\
\text { Test Used }\end{array}$ \\
\hline $\begin{array}{c}\text { Marques \& } \\
\text { González-Badillo } \\
\text { [14] }\end{array}$ & 2006 & Spain & $\begin{array}{c}\text { Not } \\
\text { mentioned }\end{array}$ & 16 & 0 & $\begin{array}{c}18 \text { to } 29 \\
\text { years old } \\
(23.1 \pm \\
4.7)\end{array}$ & $100 \%$ men & $\begin{array}{l}\text { Body mass: } 84.8 \\
\pm 13.1 \mathrm{~kg} \text {, height: } \\
1.84 .2 \pm 13.1 \mathrm{~cm}\end{array}$ & Handball & $\begin{array}{l}\text { High level } \\
\text { (international } \\
\text { and national) }\end{array}$ & $\begin{array}{l}\text { Strength and } \\
\text { power training: } \\
\text { bench press and } \\
\text { parallel squat } \\
\text { (70\% to 95\% } \\
\text { 1RM), CM and } \\
\text { sprint training }\end{array}$ & $\begin{array}{c}\text { Two to three } \\
\text { sessions per } \\
\text { week over } 12 \\
\text { weeks }\end{array}$ & $\begin{array}{l}\text { 1RM bench press, } \\
\text { 4RM parallel } \\
\text { squat, CMJ, } \\
\text { sprint tests (30m), } \\
\text { ball throwing } \\
\text { velocity }\end{array}$ & $\begin{array}{l}\text { A } \\
\text { repeated-measures } \\
\text { analysis of } \\
\text { variance with } \\
\text { Bonferroni } \\
\text { adjustment } \\
\text { was used to assess } \\
\text { gains or losses }\end{array}$ \\
\hline $\begin{array}{l}\text { Ignjatovic } \\
\text { et al. [13] }\end{array}$ & 2012 & Serbia & Randomized & 21 & 0 & $16.9 \pm 1.2$ & $\begin{array}{c}100 \% \\
\text { female }\end{array}$ & Not mentioned & Handball & Elite & $\begin{array}{l}\text { Medicine ball } \\
\text { training }\end{array}$ & $\begin{array}{c}\text { Two sessions } \\
\text { per week over } \\
12 \text { weeks }\end{array}$ & $\begin{array}{c}\text { Muscle strength: } \\
\text { 1RM bench press, } \\
\text { 1RM shoulder } \\
\text { press, for muscle } \\
\text { power: } 30 \% \text { RM } \\
\text { bench press, } 50 \% \\
\text { RM bench press, } \\
30 \% \text { RM shoulder } \\
\text { press, } 50 \% \text { RM } \\
\text { shoulder press, } \\
\text { ball throwing } \\
\text { distance }\end{array}$ & $\begin{array}{l}\text { Changes in muscle } \\
\text { power were } \\
\text { analyzed } \\
\text { separately using } 2 \\
\times 2 \text { (treatment } \times \\
\text { time) repeated } \\
\text { measure analysis } \\
\text { of variance } \\
\text { (ANOVA) }\end{array}$ \\
\hline $\begin{array}{l}\text { Carvalho } \\
\text { et al. [24] }\end{array}$ & 2014 & Portugal & $\begin{array}{c}\text { Not } \\
\text { mentioned }\end{array}$ & 12 & 0 & $\begin{array}{c}21.6 \pm \\
1.73\end{array}$ & $100 \%$ men & $\begin{array}{c}\text { Body height } 183.9 \\
\pm 0.09 \mathrm{~cm} ; \text { body } \\
\text { mass } \\
81.7 \pm 8.3 \mathrm{~kg})\end{array}$ & Handball & Semi-professional & $\begin{array}{c}\text { Strength, } \\
\text { plyometric } \\
\text { training }\end{array}$ & $\begin{array}{c}\text { Three } \\
\text { sessions per } \\
\text { week over } 12 \\
\text { weeks }\end{array}$ & $\begin{array}{c}\text { Maximum } \\
\text { dynamic and } \\
\text { isometric } \\
\text { strength, squat } \\
\text { jump, } \\
\text { countermovement } \\
\text { jump, } 40 \\
\text { consecutive } \\
\text { jumps }\end{array}$ & $\begin{array}{c}\text { A repeated } \\
\text { measurement } \\
\text { paired-samples } \\
t \text {-test was used to } \\
\text { assess the training } \\
\text { effects within } \\
\text { groups }\end{array}$ \\
\hline
\end{tabular}


Table 2. Cont

\begin{tabular}{|c|c|c|c|c|c|c|c|c|c|c|c|c|c|c|}
\hline Authors & Year & Country & $\begin{array}{c}\text { Randomized } \\
\text { or Not } \\
\text { (R, NR) }\end{array}$ & Sample & $\begin{array}{c}\text { Drop-Out } \\
\text { Rate }\end{array}$ & Age & $\begin{array}{l}\text { Gender (in } \\
\text { Percentage) }\end{array}$ & $\begin{array}{l}\text { Anthropometric } \\
\text { Features of } \\
\text { Sample (Height, } \\
\text { Body Mass, } \\
\text { BMI, etc.) }\end{array}$ & Sport & $\begin{array}{c}\text { Level } \\
\text { (International, } \\
\text { National, } \\
\text { Elite) }\end{array}$ & $\begin{array}{c}\text { Type of } \\
\text { Training }\end{array}$ & $\begin{array}{c}\text { Duration of } \\
\text { Training }\end{array}$ & $\begin{array}{l}\text { Variable } \\
\text { Measured }\end{array}$ & $\begin{array}{l}\text { Statistical } \\
\text { Test Used }\end{array}$ \\
\hline $\begin{array}{l}\text { Chelly } \\
\text { et al. [5] }\end{array}$ & 2014 & Tunisia & Randomized & 23 & 0 & $17.4 \pm 0.5$ & $100 \%$ men & $\begin{array}{c}\text { Body mass: } 79.9 \\
\pm 11.5 \mathrm{~kg}, \text { height: } \\
1.79 \pm 6.19 \mathrm{~m} \\
\text { body fat: } 13.8 \pm \\
2.1 \%\end{array}$ & Handball & Elite & $\begin{array}{l}\text { Plyometric } \\
\text { training on } \\
\text { upper and } \\
\text { lower limb }\end{array}$ & $\begin{array}{l}\text { Two sessions } \\
\text { per week over } \\
8 \text { weeks }\end{array}$ & $\begin{array}{c}\text { Force-velocity } \\
\text { test for upper } \\
\text { limbs (cycle } \\
\text { ergometer), } \\
\text { force-velocity } \\
\text { test for lower } \\
\text { limbs (cycle } \\
\text { ergometer), } \\
\text { squat jump, } \\
\text { countermovement } \\
\text { jump, throwing } \\
\text { ball test }\end{array}$ & $\begin{array}{c}\text { Training-related } \\
\text { effects were } \\
\text { assessed } \\
\text { by two-way } \\
\text { analyses of } \\
\text { variance with } \\
\text { repeated } \\
\text { measures } \\
\text { (grouped three } \\
\text { times) }\end{array}$ \\
\hline $\begin{array}{l}\text { Raeder } \\
\text { et al. [9] }\end{array}$ & 2015 & Spain & Randomized & 28 & 0 & $20.8 \pm 3.3$ & $\begin{array}{c}100 \% \\
\text { women }\end{array}$ & $\begin{array}{l}\text { Height: } 170.5 \pm \\
5.6 \mathrm{~cm}, \text { body } \\
\text { mass: } 65.2 \pm \\
8.0 \mathrm{~kg}\end{array}$ & Handball & Amateur & $\begin{array}{l}\text { Medicine ball } \\
\text { throws }\end{array}$ & $\begin{array}{c}\text { Three } \\
\text { sessions per } \\
\text { week over } 6 \\
\text { weeks }\end{array}$ & $\begin{array}{l}\text { Throwing ball } \\
\text { velocity, } \\
\text { isokinetic } \\
\text { strength: peak } \\
\text { torques of } \\
\text { shoulder } \\
\text { internal and } \\
\text { external rotators }\end{array}$ & $\begin{array}{l}\text { A two-factor } \\
\text { analysis of } \\
\text { variance for } \\
\text { repeated } \\
\text { measurements } \\
\text { was calculated to } \\
\text { determine } \\
\text { differences } \\
\text { between the } \\
\text { measurement } \\
\text { points (maineffec } \\
\text { for time), } \\
\text { between the } \\
\text { groups (main } \\
\text { effect for group), } \\
\text { and for the } \\
\text { changeover in } \\
\text { time in response } \\
\text { to the different } \\
\text { training } \\
\text { interventions } \\
\text { (three time } \\
\text { interactions) }\end{array}$ \\
\hline
\end{tabular}


Table 2. Cont.

\begin{tabular}{|c|c|c|c|c|c|c|c|c|c|c|c|c|c|c|}
\hline Authors & Year & Country & $\begin{array}{l}\text { Randomized } \\
\text { or Not } \\
\text { (R, NR) }\end{array}$ & Sample & $\begin{array}{l}\text { Drop-Out } \\
\text { Rate }\end{array}$ & Age & $\begin{array}{l}\text { Gender (in } \\
\text { Percentage) }\end{array}$ & $\begin{array}{l}\text { Anthropometric } \\
\text { Features of } \\
\text { Sample (Height, } \\
\text { Body Mass, } \\
\text { BMI, etc.) }\end{array}$ & Sport & $\begin{array}{c}\text { Level } \\
\text { (International, } \\
\text { National, } \\
\text { Elite) }\end{array}$ & Type of Training & $\begin{array}{l}\text { Duration of } \\
\text { Training }\end{array}$ & $\begin{array}{c}\text { Variable } \\
\text { Measured }\end{array}$ & $\begin{array}{l}\text { Statistical } \\
\text { Test Used }\end{array}$ \\
\hline $\begin{array}{l}\text { Genevois } \\
\text { et al. [10] }\end{array}$ & 2014 & France & Randomized & 25 & 0 & $15.8 \pm 0.8$ & $\begin{array}{c}100 \% \\
\text { women }\end{array}$ & $\begin{array}{c}\text { Height } 169.8 \pm 5 \\
\mathrm{~cm}, \text { body mass } \\
59.3 \pm 9.2 \mathrm{~kg}\end{array}$ & Handball & Elite & $\begin{array}{l}\text { Sling exercise for } \\
\text { shoulder } \\
\text { external/internal } \\
\text { rotators and } \\
\text { scapular } \\
\text { retraction }\end{array}$ & 6 weeks & $\begin{array}{l}\text { Maximal } \\
\text { throwing } \\
\text { velocity, } \\
\text { strength of } \\
\text { shoulder } \\
\text { External/internal } \\
\text { rotators and } \\
\text { scapular } \\
\text { retraction }\end{array}$ & $\begin{array}{l}\text { ANOVAs with } \\
\text { two internal } \\
\text { factors (training } \\
\text { condition and } \\
\text { training period) }\end{array}$ \\
\hline $\begin{array}{l}\text { Granados } \\
\text { et al. [26] }\end{array}$ & 2007 & Spain & Randomized & 16 & 0 & $23.1 \pm 4$ & $\begin{array}{c}100 \% \\
\text { women }\end{array}$ & $\begin{array}{c}\text { Body mass }(\mathrm{kg}) \text { : } \\
69.6 \pm 8.4 ; \text { Body } \\
\text { fat }(\%) 21.1 \pm 5.3 \\
\text { Height }(\mathrm{cm}) \\
175 \pm 6\end{array}$ & Handball & Elite & $\begin{array}{l}\text { Dynamic parallel } \\
\text { squat lift and } \\
\text { bench press and } \\
\text { power clean and } \\
\text { pullover: the load } \\
\text { in the squat lift } \\
\text { exercises (three to } \\
\text { four sets, three to } \\
\text { four reps) ranged } \\
\text { from } 60 \% \text { to } 110 \% \\
\text { of the load, in } \\
\text { parallel squat } \\
\text { actions. This } \\
\text { corresponds to a } \\
\text { load ranging } \\
\text { from } \\
\text { approximately } \\
36 \% \text { to } 77 \% 1 \text { MM } \\
\text { in the squat lift } \\
\text { exercise }\end{array}$ & $\begin{array}{l}40 \text { weeks in } \\
\text { season }\end{array}$ & $\begin{array}{c}\text { Vertical } \\
\text { jumping height, } \\
\text { throwing } \\
\text { velocity, muscle } \\
\text { power output; } \\
\text { maximal } \\
\text { strength of } \\
\text { upper and } \\
\text { lower limb }\end{array}$ & $\begin{array}{l}\text { One-way analysis } \\
\text { of variance with } \\
\text { repeated } \\
\text { measures was } \\
\text { used to determine } \\
\text { the differences } \\
\text { between tests. } \\
\text { When a } \\
\text { significant F } \\
\text { value was } \\
\text { achieved, } \\
\text { appropriate } \\
\text { Scheffe's post-hoc } \\
\text { test procedures } \\
\text { were used to } \\
\text { locate the } \\
\text { difference } \\
\text { between means }\end{array}$ \\
\hline
\end{tabular}


Table 2. Cont

\begin{tabular}{|c|c|c|c|c|c|c|c|c|c|c|c|c|c|c|}
\hline Authors & Year & Country & $\begin{array}{c}\text { Randomized } \\
\text { or Not } \\
\text { (R, NR) }\end{array}$ & Sample & $\begin{array}{l}\text { Drop-Out } \\
\text { Rate }\end{array}$ & Age & $\begin{array}{l}\text { Gender (in } \\
\text { Percentage) }\end{array}$ & $\begin{array}{l}\text { Anthropometric } \\
\text { Features of } \\
\text { Sample (Height, } \\
\text { Body Mass, } \\
\text { BMI, etc.) }\end{array}$ & Sport & $\begin{array}{c}\text { Level } \\
\text { (International, } \\
\text { National, } \\
\text { Elite) }\end{array}$ & $\begin{array}{l}\text { Type of } \\
\text { Training }\end{array}$ & $\begin{array}{l}\text { Duration of } \\
\text { Training }\end{array}$ & $\begin{array}{l}\text { Variable } \\
\text { Measured }\end{array}$ & $\begin{array}{l}\text { Statistical } \\
\text { Test Used }\end{array}$ \\
\hline $\begin{array}{l}\text { Toumi } \\
\text { et al. [19] }\end{array}$ & 2004 & France & Randomized & 22 & 0 & $21 \pm 2$ & $100 \%$ men & $\begin{array}{l}\text { Body mass }(\mathrm{kg}): \\
81 \pm 6 ; \text { Height } \\
(\mathrm{cm}): 181 \pm 8\end{array}$ & Handball & Sub-elite & $\begin{array}{c}\text { Weight training } \\
\text { group: leg press } \\
\text { machine at } 70 \% \\
\text { of maximal } \\
\text { isometric force } \\
\text { output. } \\
\text { Combined } \\
\text { training group: } \\
\text { leg press } \\
\text { machine at } 70 \% \\
\text { of maximal } \\
\text { isometric force } \\
\text { output, } \\
\text { jumping } \\
\text { exercises }\end{array}$ & $\begin{array}{c}\text { Three } \\
\text { sessions per } \\
\text { week over } 6 \\
\text { weeks }\end{array}$ & $\begin{array}{l}\text { Squat jump, } \\
\text { countermovement } \\
\text { jump, maximal } \\
\text { isometric force, } \\
\text { maximal power }\end{array}$ & $\begin{array}{l}\text { Two-way } \\
\text { ANOVA } \\
\text { (repeated } \\
\text { measures on one } \\
\text { factor) was used } \\
\text { for the statistical } \\
\text { analysis }\end{array}$ \\
\hline $\begin{array}{l}\text { van Muijen } \\
\text { et al. [20] }\end{array}$ & 1991 & Netherlands & Randomized & 56 & 11 & $23 \pm 4$ & $\begin{array}{c}100 \% \\
\text { women }\end{array}$ & $\begin{array}{c}\text { Body mass }(\mathrm{kg}) \text { : } \\
65.3 \pm 5.7 ; \text { Body } \\
\text { fat }(\%) 27.5 \pm 2.9 ; \\
\text { Height }(\mathrm{cm}): \\
169.5 \pm 5.7\end{array}$ & Handball & Sub-elite & $\begin{array}{l}\text { Group 1: } \\
\text { training with } \\
\text { normal hand } \\
\text { balls }(400 \mathrm{~g}) \text {. } \\
\text { Group 2: } \\
\text { training with } \\
\text { heavy balls } \\
\text { (500 g). Group } \\
\text { 3: training with } \\
\text { light balls } \\
(300 \mathrm{~g}) .\end{array}$ & $\begin{array}{l}\text { Two sessions } \\
\text { per week over } \\
8 \text { weeks }\end{array}$ & $\begin{array}{l}\text { Throwing ball } \\
\text { velocity, } \\
\text { maximal } \\
\text { isokinetic torque } \\
\text { of elbow } \\
\text { extensors and } \\
\text { medial shoulder } \\
\text { rotators }\end{array}$ & Student's $t$-test \\
\hline
\end{tabular}




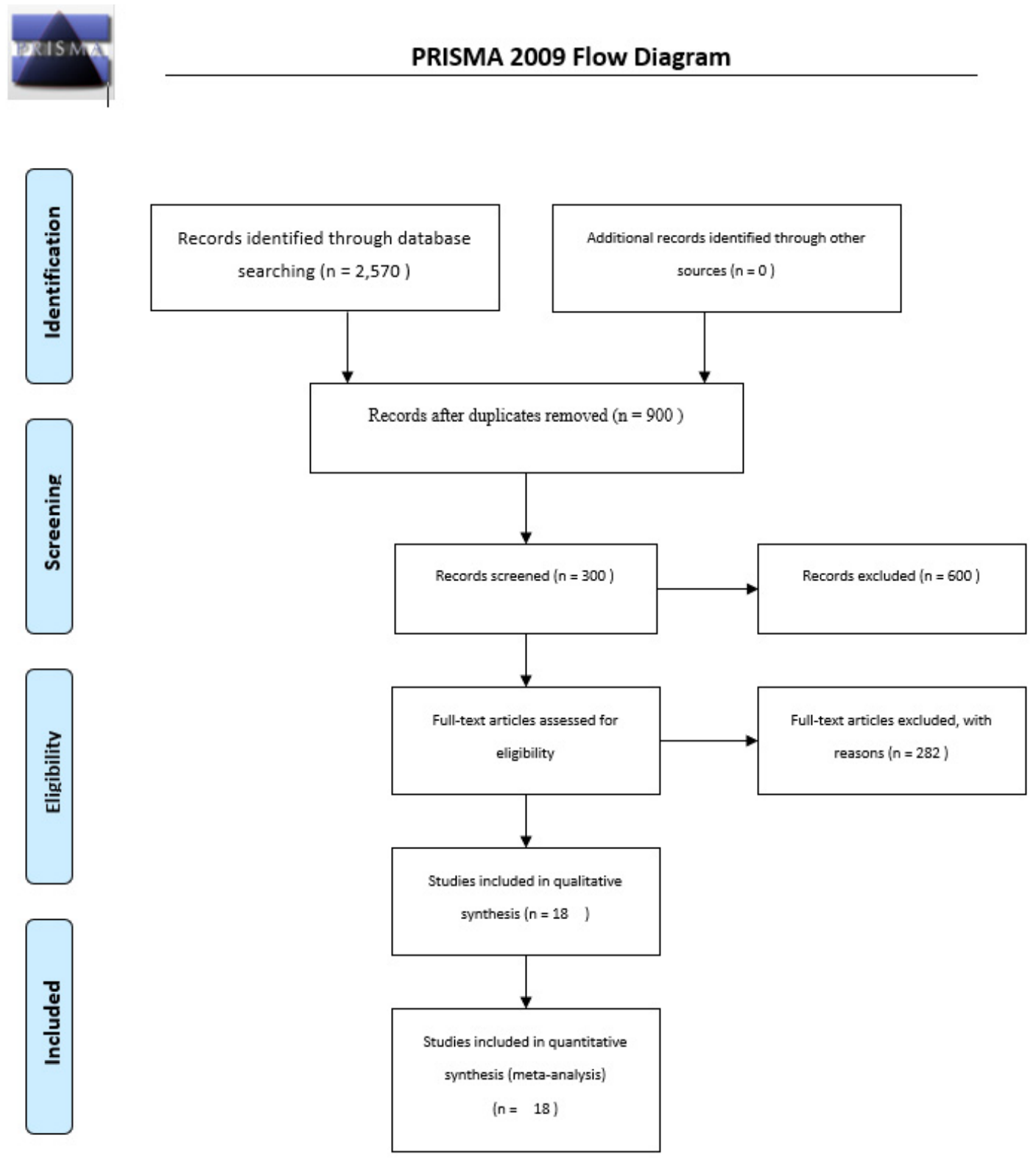

Figure 1. The process of studies retrieval and inclusion adopted in the present systematic review and meta-analysis.

\subsection{Meta-Analysis}

The overall ES was 0.996 ([95\%CI 0.827-1.165], $p=0.0000 ; \mathrm{k}$ studies $=82$ ). Due to the significant amount of heterogeneity $\left(\mathrm{I}^{2}=82.73 \%\right)$, the random-effect model was carried out. At the multivariate meta-regression $\left(\operatorname{tau}^{2}=0.19\right.$, tau $\left.=0.44, \mathrm{I}^{2}=65.55 \%, \mathrm{Q}=191.57, \mathrm{df}=66, p=0.0000\right)$, the effect of publication year was significant $(p=0.0005$; regression coefficient $=0.0007$ [95\%CI 0.03-0.11], SE $=$ $0.02, \mathrm{z}=3.46)$, as well as the effect of country ( $p=0.0000$; regression coefficient $=-1.08[95 \% \mathrm{CI}-1.43$ to -0.73 ] for Europe versus Africa, $\mathrm{SE}=0.18, \mathrm{z}=-6.06)$, gender $(p=0.0022$; regression coefficient $=-0.01$ [95\%CI -0.01 to -0.00$], \mathrm{SE}=0.00, \mathrm{z}=-3.06)$, and expertise level $(p=0.0346$; regression coefficient $=$ $0.92[95 \% \mathrm{CI} 0.07-1.18]$ for sub-elite versus elite athletes, $\mathrm{SE}=0.44, \mathrm{z}=2.11$ ). The number of weeks was statistically borderline $(p=0.0655$; regression coefficient $=0.08$ [ $95 \% \mathrm{CI}-0.01$ to 0.17$], \mathrm{SE}=0.04, \mathrm{z}=$ $1.84)$, whereas the number of sessions per week was not significant $(p=0.4233)$. The other parameters failed to reach the significance threshold (age $p=0.3233$ ). There was a significant difference among the outcome selected $(\mathrm{Q}=19.63, \mathrm{df}=4, p=0.0006$; for isometric strength, regression coefficient $=$ 0.30 [95\%CI $-0.40-0.99], \mathrm{SE}=0.35, \mathrm{z}=0.84, p=0.4018$; for maximal strength, regression coefficient $=$ 
1.11 [95\%CI 0.45-1.76], $\mathrm{SE}=0.33, \mathrm{z}=3.31, p=0.0009$; for power, regression coefficient $=0.30[95 \% \mathrm{CI}$ $-0.16-0.76], \mathrm{SE}=0.23, \mathrm{z}=3.13, p=0.1994$; for throwing, regression coefficient $=0.81$ [95\%CI $0.30-1.32]$, $\mathrm{SE}=0.26, \mathrm{z}=3.13, p=0.0017)$. There was evidence of publication bias, both in visually inspecting the Funnel plot and carrying out Egger's linear regression test (intercept $=5.27$ [95\%CI 4.74-5.81], SE = $0.27, \mathrm{t}=19.58, p=0.0000$ ). Moreover, with the Duval and Tweedie trim-and-fill analysis, 30 studies were trimmed (thus resulting in a "true ES" of 0.378 [95\%CI 0.184-0.571], $\mathrm{Q}=928.17$ ).

When stratifying according to expertise level, ES was higher among elite athletes (ES 1.067 [95\%CI $0.882-1.251], p=0.000$ ) than among sub-elite athletes (ES 0.510 [95\%CI 0.136-0.883], $p=0.008 ; \mathrm{I}^{2}=$ 67.38, random-effect model). Among elite athletes, there was an evidence of publication bias. Among sub-elite athlete studies, there was a small amount of evidence of publication bias (intercept $=7.40$ [95\%CI 4.99-9.80], $\mathrm{t}=7.28, p=0.00017)$ : one study was trimmed, resulting into a "true ES" of 0.350 ([95\%CI -0.114-0.813], Q = 43.29).

When stratifying according to gender, ES was lower among female athletes (ES 0.783 ([95\%CI $0.435-1.130], p=0.000$ ), than among male athletes (ES 1.042 ([95\%CI 0.854-1.230], $p=0.000$ ).

The impact of RT on isokinetic strength (Figure 2) was not statistically significant (ES 0.079 [95\%CI $-0.060-0.219], p=0.265)$.

\begin{tabular}{|c|c|c|c|c|c|c|c|}
\hline \multirow[t]{2}{*}{ Study name } & \multicolumn{7}{|c|}{ Statistics for each study } \\
\hline & $\begin{array}{c}\text { Hedges's } \\
\text { g }\end{array}$ & $\begin{array}{l}\text { Standard } \\
\text { error }\end{array}$ & Variance & $\begin{array}{c}\text { Lower } \\
\text { limit }\end{array}$ & $\begin{array}{c}\text { Upper } \\
\text { limit }\end{array}$ & ZValue & p-Value \\
\hline Holm et al., 2003e & 0.080 & 0.165 & 0.027 & -0.244 & 0.404 & 0.484 & 0.629 \\
\hline Holm et al., $2003 \mathrm{f}$ & 0.190 & 0.165 & 0.027 & -0.134 & 0.514 & 1.149 & 0.251 \\
\hline Holm et al., $2003 \mathrm{~g}$ & .0 .080 & 0.163 & 0.027 & -0.399 & 0.239 & -0.491 & 0.623 \\
\hline Holm et al., 2003h & -0.100 & 0.165 & 0.027 & -0.424 & 0.224 & -0.605 & 0.545 \\
\hline Caravalho et al., $2014 \mathrm{f}$ & 0.620 & 0.304 & 0.092 & 0.025 & 1.215 & 2.041 & 0.041 \\
\hline Caravalho et al., $2014 \mathrm{~g}$ & 0.370 & 0.282 & 0.080 & -0.183 & 0.923 & 1.312 & 0.190 \\
\hline Caravalho et al., $2014 \mathrm{~h}$ & 0.110 & 0.272 & 0.074 & -0.424 & 0.644 & 0.404 & 0.686 \\
\hline \multirow[t]{2}{*}{ Caravalho et al., 2014i } & -0.030 & 0.277 & 0.077 & -0.573 & 0.513 & -0.108 & 0.914 \\
\hline & 0.079 & 0.071 & 0.005 & -0.060 & 0.219 & 1.115 & 0.265 \\
\hline
\end{tabular}

Figure 2. The impact of resistance training on isokinetic strength among handball players.

The impact of RT on throwing (Figure 3) (ES 1.360 [95\%CI 0.992-1.728], $p=0.000$ ) was significant.

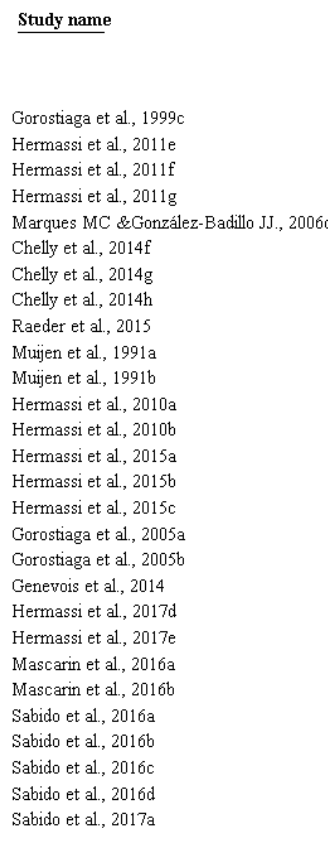

\begin{tabular}{|c|c|c|c|c|c|c|}
\hline & & & & tudy & & \\
\hline $\begin{array}{l}\text { Hedges's } \\
\text { g }\end{array}$ & $\begin{array}{l}\text { Standard } \\
\text { error }\end{array}$ & Variance & $\begin{array}{c}\text { Lower } \\
\text { limit }\end{array}$ & $\begin{array}{c}\text { Upper } \\
\text { limnit }\end{array}$ & Z-Value & p-Val \\
\hline 0,300 & 0,314 & 0.098 & -0.315 & 0.915 & 0.956 & \\
\hline 1,550 & 0,432 & 0.186 & 0.704 & 2.396 & 3.592 & 000 \\
\hline 2,510 & & & 1.324 & 3.696 & & 000 \\
\hline 2,310 & 0,583 & 0.340 & 1.166 & 3.454 & 3.959 & 000 \\
\hline 0,730 & & 75 & 93 & 1.267 & 2662 & 008 \\
\hline 2,440 & 593 & 52 & 1.278 & 3.602 & 4.114 & 0000 \\
\hline 3,420 & & & 1.880 & 4.960 & 4.351 & 000 \\
\hline 1,700 & & & 0.797 & 2.603 & 3692 & 000 \\
\hline 2,820 & 91 & 849 & 1.662 & 3.978 & 4.774 & \\
\hline$-0,180$ & & & -0.668 & 0.308 & -0.723 & 470 \\
\hline 0,260 & & & -0.228 & 0.748 & .044 & 296 \\
\hline 4,600 & & 30 & 2.256 & 6.944 & 847 & 000 \\
\hline 3,280 & & & 20 & 5.240 & 280 & 001 \\
\hline 2,280 & 0 & 42 & 0.976 & 3584 & 3.428 & 00 \\
\hline 2,290 & & 33 & 1.000 & 3.580 & 480 & .0 \\
\hline 2,960 & 814 & 662 & 1.365 & 4.555 & 3.638 & .000 \\
\hline & & & & 1.265 & 508 & 01. \\
\hline 0,780 & 0,288 & 183 & 0.215 & 1.345 & 2.708 & 007 \\
\hline 0,100 & & & -0.285 & 0.485 & 0.509 & 61 \\
\hline 1,550 & & & 0.704 & 2.396 & 3.592 & 00 \\
\hline 2,510 & & & 1.324 & 3.696 & 4.148 & 000 \\
\hline & & & 1.349 & 2911 & 5.348 & \\
\hline 2,810 & 0,492 & 242 & 1.845 & 3775 & 5.708 & .000 \\
\hline 0,400 & & & -0.167 & 0.967 & 1.383 & 167 \\
\hline 0,320 & & & -0.233 & 0.873 & 1.134 & 257 \\
\hline 0,380 & & & -0.173 & 0.933 & 1.347 & 178 \\
\hline 0,260 & & & -0.283 & 0.803 & 0.938 & 0.3 \\
\hline$-0,190$ & & 0.084 & -0.758 & 0.378 & -0.655 & \\
\hline 1,360 & 0,188 & 0.035 & 0.992 & 1.728 & 7.245 & 0.00 \\
\hline
\end{tabular}

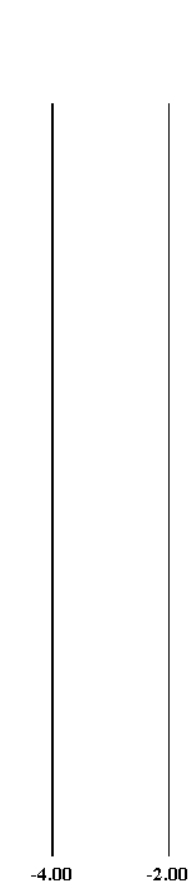

Hedges's g and $95 \% \mathrm{Cl}$

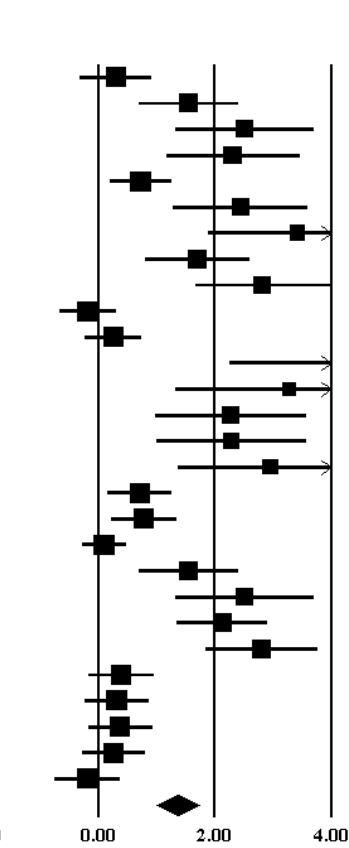

Figure 3. The impact of resistance training on throwing among handball players. 
Moreover, the impact of RT on isometric strength (Figure 4) (ES 0.398 [95\%CI 0.096-0.700], $p=$ 0.010), on maximal strength (Figure 5) (ES 1.824 [95\%CI 1.305-2.343], $p=0.000$ ), and on power (Figure 6 ) (ES 0.892 [95\%CI 0.656-1.128], $p=0.000$ ) was significant.

\begin{tabular}{|c|c|c|c|c|c|c|c|}
\hline \multirow[t]{2}{*}{ Study name } & \multicolumn{7}{|c|}{ Statistics for each study } \\
\hline & $\begin{array}{c}\text { Hedges's } \\
\underline{\underline{g}}\end{array}$ & $\begin{array}{c}\text { Standard } \\
\text { error }\end{array}$ & Variance & $\begin{array}{c}\text { Lower } \\
\text { limit }\end{array}$ & $\begin{array}{c}\text { Upper } \\
\text { limit }\end{array}$ & Z-Value & p-Value \\
\hline Gorostiaga et al., $1999 \mathrm{e}$ & 0.700 & 0.363 & 0.131 & -0.011 & 1.411 & 1.931 & 0.053 \\
\hline Gorostiaga et al., 1999f & 0.580 & 0.344 & 0.119 & -0.095 & 1.255 & 1.684 & 0.092 \\
\hline Caravalho et al., 2014d & 0.150 & 0.272 & 0.074 & -0.384 & 0.684 & 0.551 & 0.582 \\
\hline Caravalho et al., 2014e & 0.360 & 0.282 & 0.080 & -0.193 & 0.913 & 1.276 & 0.202 \\
\hline & 0.398 & 0.154 & 0.024 & 0.096 & 0.700 & 2.584 & 0.010 \\
\hline
\end{tabular}

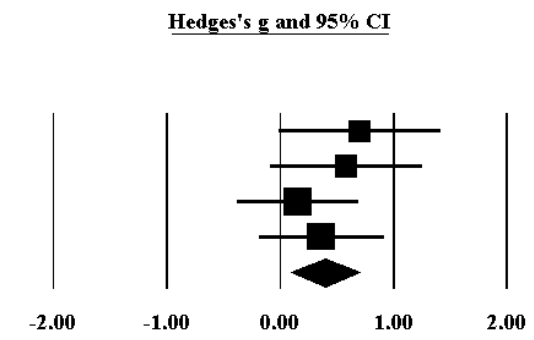

Figure 4. The impact of resistance training on isometric strength among handball players.

\begin{tabular}{|c|c|c|c|c|c|c|c|}
\hline \multirow[t]{2}{*}{ Study name } & \multicolumn{7}{|c|}{ Statistics for each study } \\
\hline & $\begin{array}{l}\text { Iedges's } \\
\text { g }\end{array}$ & $\begin{array}{c}\text { Standard } \\
\text { error }\end{array}$ & Variance & $\begin{array}{c}\text { Lower } \\
\text { limit }\end{array}$ & $\begin{array}{c}\text { Upper } \\
\text { limit }\end{array}$ & Z-Value & p-Value \\
\hline Gorostiaga et al., $1999 \mathrm{~d}$ & 0.710 & 0.359 & 0.129 & 0.007 & 1.413 & 1.980 & 0.048 \\
\hline Hermassi et al. $2011 \mathrm{~d}$ & 1.570 & 0.434 & 0.188 & 0.719 & 2.421 & 3.618 & 0.000 \\
\hline Marques MC \&González-Eadillo JJ., 2006b & b. 2.280 & 0.485 & 0.235 & 1.330 & 3.230 & 4.703 & 0.000 \\
\hline Harmmami et al, $2018 \mathrm{~d}$ & 2.130 & 0.491 & 0.241 & 1.167 & 3.093 & 4.335 & 0.000 \\
\hline Hermassi et al. $2017 \mathrm{a}$ & 2.510 & 0.605 & 0.366 & 1.324 & 3.696 & 4.148 & 0.000 \\
\hline Hermassi et.al., $2017 \mathrm{f}$ & 2.640 & 0.629 & 0.396 & 1.407 & 3.873 & 4.195 & 0.000 \\
\hline Hermassi et al, $2017 \mathrm{i}$ & 2.360 & 0.583 & 0.340 & 1.216 & 3.504 & 4.045 & 0.000 \\
\hline Sabido et al., $2017 \mathrm{c}$ & 1.190 & 0.396 & 0.156 & 0.415 & 1.965 & 3.009 & 0.003 \\
\hline & 1.824 & 0.265 & 0.070 & 1.305 & 2.343 & 6.890 & 0.000 \\
\hline
\end{tabular}

Hedges's g and $95 \% \mathrm{CT}$

Figure 5. The impact of resistance training on maximal strength among handball players.

$\underline{\text { Study name }}$

Holm et a1., 2003a

Holm et. al., 2003b

Holm et. al., 20030

Holm et al., 2003d

Gorostiaga et. al., 1999 a

Gorostiaga et. al., 1990

Hermassi et al., $2011 \mathrm{a}$

Hermassi et al., 2011b

Hermassi et al., $2011 \mathrm{c}$

Marques \&Gorzalez-Badillo, 2006:

Caravalho et al, $2014 \mathrm{a}$

Caravallho et al, 2014b

Caravalho et al, 2014c

Chelly et al., $2014 a$

Chelly et al, $2014 \mathrm{c}$

Chelly et al, 2014e

Hermassi et al., $2014 a$

Hermassi et al, 2014c

Gorostiaga et. al., 20050

Antoritio et al, 2017 a

Antortio et al, $2017 \mathrm{~b}$

Hammami et al., 2018a

Hammami et al., 20181

Hammanni et al., $2018 \mathrm{c}$

Hermassi et al, 2017b

Hermassi et al., $2017 \mathrm{c}$

Hermassi et a1., $2017 \mathrm{~g}$

Hermassi et al., 2017h

Hermassi et al., $2017 \mathrm{j}$

Hermassi et al., $2017 \mathrm{k}$

Sabido et al. , $2017 \mathrm{~b}$

Sabido et al, , 2017d

Sabido et al, 2017 e

\begin{tabular}{|c|c|c|c|c|c|c|}
\hline & & & & & & \\
\hline $\begin{array}{c}\text { Hedges's } \\
\mathrm{g}\end{array}$ & $\begin{array}{l}\text { Standard } \\
\text { error }\end{array}$ & Variance & $\begin{array}{r}\text { Lower } \\
\text { limit }\end{array}$ & $\begin{array}{l}\text { Lpper } \\
\text { limit }\end{array}$ & Z-Value $\mathrm{p}$ & p-Value \\
\hline $\begin{array}{l}0.460 \\
0.240\end{array}$ & & $\begin{array}{l}0.031 \\
0.027\end{array}$ & $\begin{array}{r}0.116 \\
-10.084\end{array}$ & $\begin{array}{l}0.804 \\
0.564\end{array}$ & $\begin{array}{l}2.623 \\
1.451\end{array}$ & \\
\hline $\begin{array}{l}-0.020 \\
-\end{array}$ & 163 & 0.027 & $\begin{array}{l}-0.339 \\
\end{array}$ & 0.299 & -0.123 & 0.902 \\
\hline 0.130 & & & -0.229 & 0.489 & & 0.477 \\
\hline 0.300 & 316 & 0.100 & -0.319 & 0.919 & 0.949 & 0.343 \\
\hline 0.290 & & & -0.329 & 0.909 & 0.918 & 0.359 \\
\hline 0.620 & & & -0.004 & & & 051 \\
\hline 0.920 & & & 0.254 & & & \\
\hline 0.130 & & & -0.418 & 0.678 & & 0.642 \\
\hline & & & & & & \\
\hline & & & -0.263 & & & \\
\hline 160 & & & -0.374 & 0.6 & & 0.557 \\
\hline a. & & & -0.198 & & & 206 \\
\hline 0. & & & -0.021 & & & 0.059 \\
\hline 0. & & & -0.1 & & & \\
\hline 1 & & & & & & 003 \\
\hline 1. & & & & 2.0 & & 0001 \\
\hline & & & -0.001 & 1.181 & & \\
\hline & & & & 6.741 & & 0.000 \\
\hline a. & & & -0.007 & 0.787 & 925 & 0.054 \\
\hline 660 & & & 0.1 & 1.162 & 2.579 & 0.010 \\
\hline & & & & 1.1 & & 0.029 \\
\hline & & & 2.040 & 4.900 & & 0.000 \\
\hline & & & 1.9 & 4.7 & 749 & 0.000 \\
\hline & & & 1.377 & 3.503 & & 0.000 \\
\hline & & & & & & 0.000 \\
\hline & & & 1.980 & 5.260 & & 0.000 \\
\hline 3.470 & & 0.620 & 1.915 & 5.025 & 4.375 & 0.000 \\
\hline & & & & & & \\
\hline & & & 1.464 & & 400 & 0.00 \\
\hline 3.760 & & 0.7 & 2.097 & 5.423 & 4.431 & 0.000 \\
\hline & & & -0.137 & & & \\
\hline & & & -0.268 & 0.868 & 1.034 & \\
\hline & & 0.108 & 0.086 & 1.374 & 2.223 & 0.026 \\
\hline 0.892 & 0.121 & 0.015 & 0.656 & 1.128 & 7.398 & 0.000 \\
\hline
\end{tabular}

Hedges's g and $95 \% \mathrm{CI}$

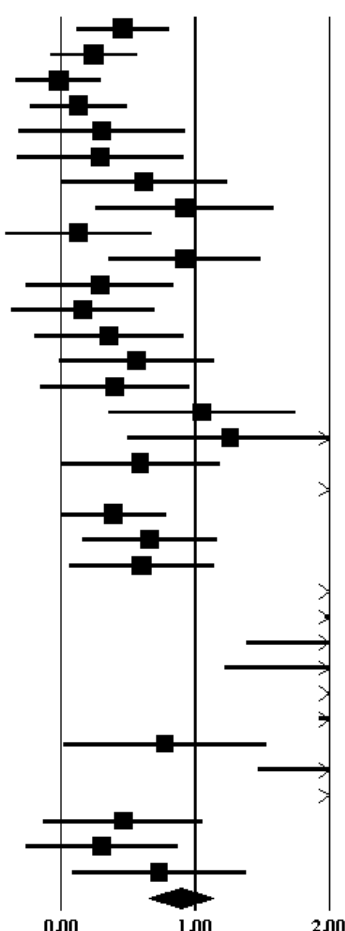

Figure 6. The impact of resistance training on muscle power among handball players.

Statistically significant determinants of the impact on power were the country, gender and number of weeks, whilst determinants of the impact on throwing were publication year, country, and times 
of sessions per week. In all other cases, it was not possible to perform meta-regressions due to the insufficient number of available studies per covariate or due to multi-collinearity issues.

Considering the control groups, the overall ES was 0.173 ([95\%CI 0.048-0.299], $p=0.007$ ); 0.071 ([95\%CI $-0.176-0.319], p=0.573$ ) for throwing, 0.266 ([95\%CI 0.144-0.388], $p=0.000$ ) for power, 0.138 ([95\%CI $-0.188-463], p=0.407)$ for maximal strength, and 0.163 [95\%CI $-0.274-0.600], p=0.466$ ) for isometric strength.

Quality assessment of the studies is reported in Supplementary Material Table S1.

\section{Discussion}

\subsection{Isokinetic Strength}

The most significant gains can usually be verified in isometric and eccentric force production, mainly in the knee flexors [23]. These improvements can be justified by the fact that these actions are not performed with such a frequency as concentric actions, so they are more susceptible to training stimuli. Moreover, handball is a game characterized by complex movements that require constant mobility of knee extensors in order to achieve high-speed and quick changes of direction [6-8,26,27].

Since the knee flexors are not involved, their strength is commonly relatively low, which enables players to obtain greater strength gains after the training program. Gorostiaga et al. [15], for instance, found gains of $13 \%$ and $9 \%$ in leg extensor and flexor muscles, respectively, after a 6-week period of heavy training. Similarly, Carvalho et al. [24] showed an improvement in the peak torque of thigh muscles and concentric ratio after a 4-week heavy training program. Likewise, most strength variables increased between the two moments of assessment; however, the most significant differences were found with the knee isometric left antagonist $(11.4 \%)$, eccentric left agonist $(9.6 \%)$, isometric right agonist $(8 \%)$ and eccentric right antagonist (6.6\%).

\subsection{Throwing Ball Velocity}

Elite handball players achieve significantly higher throwing ball velocities than their lower level counterparts [28,29] - with an 8-9\% advantage in elite men [15] and 10-11\% advantage in elite females [26]. Hermassi et al. [7] and Gorostiaga et al. [15] noted an increase in all types of throw ball velocities (standing throw, three-step running throw and jump-throw) following 8 weeks of heavy RT. Similar improvements were obtained after 8-week bi-weekly plyometric training [5] and after an 8-week tri-weekly upper limb specific RT in handball players [25].

By increasing workloads, introducing higher throwing weights (pyramid training), accompanied with a relatively small number of repetitions and less training time, is required to obtain an optimal response. Gorostiaga et al. [15] noted a significant enhancement $(p<0.001)$ of standing throwing ball after 6 weeks of heavy upper limb RT programs. Moreover, van den Tillaar [29], comparing the benefits of various handball-training programs, found that RT was the most effective in increasing throwing ball velocity.

However, existing studies are mainly focused on concentric exercises [28,29], even though most actions required during handball imply a combination of eccentric and concentric muscular contractions, involving, for example, both concentric and eccentric phases of squat jumps [28,29].

Different studies [30] reported a significant increase by $1.2-18 \%$ in throwing ball velocity after RT (three sets of six repetitions with a load of around $85 \%$ of $1 \mathrm{RM}$ ), with the training groups performing extra training compared to the control groups.

A combination of muscle strength, handball techniques, and competitive skills training can significantly enhance maximal and specific explosive strength of the upper limb over an 8- to 10-week training program $[6-8,12,13,17,28]$. This increase should confer players an advantage in sustaining the muscle contractions required during ball throwing, hitting, blocking, pushing, and holding $[6,7,15,17]$. 
In conclusion, throwing ball velocity can be increased positively after RT programs that incorporate, as a minimum, training two to three times per week for 5 weeks of general RT for the upper body or specific resistance protocols.

\subsection{Isometric Strength}

Gorostiaga et al. [17] showed that during the 6-week training period, the mean 1RM performance in the training group improved in the leg extensor muscles (leg press) from $126.7 \mathrm{~kg}$ to $142.2 \mathrm{~kg}$ $(p<0.01)$, and in the upper extremity muscles (pec-deck) from $36.1 \mathrm{~kg}$ to $44.4 \mathrm{~kg}(p<0.01)$. This increase occurred mainly during the first 2 weeks, whereas there was only a slight improvement during the following 4 weeks of training. The addition of a 6-week period of heavy training resulted in considerable gains in the maximal muscle strength of the leg extensors $(13 \%)$ and the arm muscles $(23 \%)$, with minor changes in the maximal isometric force of the leg flexor muscles $(9 \%)$.

A difference in strength gains between upper and lower extremity muscles can be explained by the difference in initial conditioning between knee extensor and upper body muscles, depending also on the pattern of quantity and/or intensity of daily physical use.

Interestingly, studies investigating the effects of concurrent strength and endurance training found an interference in the optimal development of muscle strength [29]. Handball training sessions and competitive games place energy production demands on the leg muscles mainly through aerobic processes.

\subsection{Maximal Strength}

Competitive performance in handball depends not only on muscle strength, but also on the ability to exert force at the speed required by this discipline. The longer contraction durations appear to be best suited for maximizing muscle strength [29]. The training programs developed for strength/power for handball players have traditionally focused on resistance exercises (i.e., squat, pullover and bench press) that primarily emphasize maximal force production $[17,29]$.

These exercises, despite an initial explosive contraction, are performed at a slow velocity of movement and have the greatest effect on strength improvement at the slow velocity/high force segment of the force-velocity-power curve [12,13]. However, this mode of training does not appear to maximize power performance, especially in the experienced RT players.

In this context, Gorostiaga et al. [17] found that RT can improve the strength of both the upper extremity muscles $(23 \% ; p<0.01)$ and the leg extensors $(12.2 \% ; p<0.01)$, although no gains can be anticipated from low resistance forms of activity such as normal handball practice. Furthermore, Hermassi et al. [30] and Chelly et al. [5] reported gains for the upper and lower limbs (21.8 and 14.5\%, for bench press and half-squat, respectively) even larger than those observed by Gorostiaga et al. [17] and Hermassi et al. [7], possibly due to differences in either the initial training status of the players and/or the training exercises performed.

The results of Hermassi et al. [6-8] are in agreement with other investigations [28] of plyometric training for the upper and lower limbs. The increased workload positively transferred to throws with a regular handball ball. According to Hermassi et al. [30], a combination of strength, handball technique, and competitive skills training significantly enhanced the maximal and specific explosive strength of the upper extremities over the 10-week program. The increase in maximal upper limb and lower limb maximal strength should give players an advantage in sustaining the forceful muscle contractions required during such actions [17].

On the other hand, Gorostiaga et al. [15] studied the effect of an entire season of play (45 weeks) on the strength-load relationships for the arm extensor muscles of elite male handball players. Performance was assessed on four occasions: the beginning (T1) of the first preparatory period, at the beginning (T2) and the end (T3) of the first competitive period, and at the end of the second competitive period (T4). Training was periodized from a high-volume, low-intensity phase during the preparatory period to a low-volume, high-intensity phase toward the competitive period. Values of $1 \mathrm{RM}$ bench 
press obtained at T3 increased significantly $(p<0.01)$ compared with T1. The greater number of weekly RT sessions could explain the higher increase in 1RM upper limb strength in this study.

Marques et al. [12] examined the effects of 12 weeks of RT (two to three sessions per week) in high-level handball players with loads ranging from $70-85 \%$ of concentric $1 \mathrm{RM}$ bench press. They noted a $28 \%$ increase in $1 \mathrm{RM}$ bench press; however, in a similar study by Hermassi et al. [30] the training group improved their 1RM bench press by only $16 \%$. Whether assessed by $1 \mathrm{RM}$ pullover or bench press, moderate strength training showed gains of $24 \%$ and $6 \%$ for $1 \mathrm{RM}$ pullover and $1 \mathrm{RM}$ bench press, respectively [30]. However, such gains did not statistically surpass the gains seen in those following the control regimen, and they were significantly less than those in a similar study by other researchers [28].

\subsection{Muscle Power}

Few investigations in team handball examined the effects of RT using dynamic exercises on the peak muscle power $\left(\mathrm{W}_{\text {peak }}\right)$ determined by means of a cycling force-velocity test $[6,7]$. For instance, Hermassi et al. [6] compared gains of muscle peak power, using successive eccentric-concentric exercises for the upper and lower body. Gains of absolute power for both the lower $(18 \% ; p<0.01)$ and upper $(13 \% ; p<0.01)$ extremities [7] were reported, in absence of significant changes in relative power to body mass for the upper limbs.

The prescribed loads during RT enhance muscle power and thus sport performance [5,29], as also shown by Hermassietal. [6], initiating feedback reflexes from the Golgi tendon organs and/or improving the synchronization of the firing motor unit [15].

Moreover, Hermassi et al. [6] investigated elite handball players participating in a 12-week in-season strength training program, with a frequency of two sessions per week (two exercises for the upper limbs, like pullover and bench press, and for the lower limbs, such as half squat). Loads were $80-95 \%$ of the personal 1RM, based on a succession of eccentric-concentric muscle contractions at a slow velocity, interspersed by rest intervals of 3-4 min between repetitions. Heavy training led to improvements in both absolute $(\mathrm{W})(12.39 \% ; p<0.01)$ and relative $(\mathrm{W} / \mathrm{kg})(12.9 \% ; p<0.01)$ muscle power for the upper limbs, suggesting that muscle power gain could be attributable to an increase in the regional muscle volume of the upper limbs [6].

In fact, the average percentage increase in muscle power per unit of muscle volume (W/l) for the heavy training group ( $8.5 \%)$ tended to be higher than for controls $(3.7 \%)$. Heavy RT may increase muscle bulk appreciably, potentially due to neuronal adaptation [29].

In fact, 12 weeks of heavy RT led to a considerable gain in muscle power $(\mathrm{W})(12.41 \% ; p<0.001)$ and in the relative power $(\mathrm{W} / \mathrm{kg})(13.1 \% ; p<0.01)$ of the upper limbs, but no changes when power was expressed per liter of upper limb muscle volume (W/l) [6]. The average percentage increase in muscle power per unit of muscle volume (W/l) for the heavy training group $(5.1 \pm 6 \%)$ was higher than for the control group $(0.5 \pm 9.7 \%)$. The average percentage increase in muscle power per unit of muscle volume $(\mathrm{W} / \mathrm{l}$ of the thigh) for the heavy training group $(3.5 \pm 4.4 \%)$ was higher than for the control group $(0.1 \pm 3.2 \%)$.

Although the load prescription based upon the maximizing of mechanical power output appears an attractive strategy to enhance the muscle power of the limbs, performance may be critically dependent on the ability to exert force at speeds specific to a given athletic discipline. Longer contraction durations have been associated with heavier loads, so the prescription of such loads would seem best suited to maximizing strength.

\subsection{Strengths and Limitations}

To the best of our knowledge, this is the first systematic review and meta-analysis that comprehensively and quantitatively assesses the impact of RT among handball players. However, despite its novelty, the present investigation suffers from a number of shortcomings which should be 
properly recognized and addressed. The main drawbacks are represented by the significant amount of heterogeneity and the evidence of publication bias that is present in many fields of the research domain.

\section{Conclusions}

Our systematic review and meta-analysis showed that RT has a significant impact in handball players in terms of maximal strength (isokinetic and isometric strength), muscle power of both upper and lower limbs, and throwing velocity. The present review has important practical implications, in that handball coaches could design conditioning protocols based on our results. However, due to the above-mentioned shortcomings, including evidence of bias and high, statistically significant heterogeneity among studies, further high-quality investigations in the field are urgently needed.

Supplementary Materials: The following are available online at http://www.mdpi.com/1660-4601/17/8/2663/s1, Table S1: Quality assessment of studies included in the present systematic review and meta-analysis.

Author Contributions: Conceptualization, All authors.; methodology, All authors; software, N.L.B. and M.R..; validation, N.L.B., M.R. and K.C.; formal analysis, N.L.B., M.R. and K.C.; investigation, N.L.B. and M.R.; resources, N.L.B. and M.R.; data curation, N.L.B., M.R. and K.C.; writing-Original draft preparation, All authors; writing-Review and editing, All authors; visualization, All authors; supervision, N.L.B. and K.C.; project administration, N.L.B. and M.R.; funding acquisition, N.L.B. All authors have read and agreed to the published version of the manuscript.

Funding: This research received no external funding.

Conflicts of Interest: The authors declare no conflicts of interest.

\section{References}

1. Black, G.M.; Gabbett, T.J.; Cole, M.H.; Naughton, G. Monitoring Workload in Throwing-Dominant Sports: A Systematic Review. Sports Med. 2016, 46, 1503-1516. [CrossRef] [PubMed]

2. Karcher, C.; Buchheit, M. On-court demands of elite handball, with special reference to playing positions. Sports Med. 2014, 44, 797-814. [CrossRef] [PubMed]

3. Wagner, H.; Finkenzeller, T.; Würth, S.; von Duvillard, S.P. Individual and team performance in team-handball: A review. J. Sports Sci. Med. 2014, 13, 808-816. [PubMed]

4. Manchado, C.; Tortosa-Martínez, J.; Vila, H.; Ferragut, C.; Platen, P. Performance factors in women's team handball: Physical and physiological aspects-a review. J. Strength Cond. Res. 2013, 27, 1708-1719. [CrossRef] [PubMed]

5. Chelly, M.S.; Hermassi, S.; Aouadi, R.; Shephard, R.J. Effects of 8-week in-season plyometric training on upper and lower limb performance of elite adolescent handball players. J. Strength Cond. Res. 2014, 28, 1401-1410. [CrossRef] [PubMed]

6. Hermassi, S.; Chelly, M.S.; Fathloun, M.; Shephard, R.J. The effect of heavy- vs. moderate-load training on the development of strength, power, and throwing ball velocity in male handball players. J. Strength Cond. Res. 2010, 24, 2408-2418. [CrossRef]

7. Hermassi, S.; Chelly, M.S.; Tabka, Z.; Shephard, R.J.; Chamari, K. Effects of 8-week in-season upper and lower limb heavy resistance training on the peak power, throwing velocity, and sprint performance of elite male handball players. J. Strength Cond. Res. 2011, 25, 2424-2433. [CrossRef]

8. Hermassi, S.; Gabbett, T.J.; Ingebrigtsen, J.; van den Tillaar, R.; Chelly, M.S.; Chamari, K. Effects of a Short-Term In-Season Plyometric Training Program on Repeated- Sprint Ability, Leg Power and Jump Performance of Elite Handball Players. Int. J. Sports Sci. Coach. 2014, 9, 1205-1216. [CrossRef]

9. Raeder, C.; Fernandez-Fernandez, J.; Ferrauti, A. Effects of Six Weeks of Medicine Ball Training on Throwing Velocity, Throwing Precision, and Isokinetic Strength of Shoulder Rotators in Female Handball Players. J. Strength Cond. Res. 2015, 29, 1904-1914. [CrossRef]

10. Genevois, C.; Berthier, P.; Guidou, V.; Muller, F.; Thiebault, B.; Rogowski, I. Effects of 6-week sling-based training of the external-rotator muscles on the shoulder profile in elite female high school handball players. J. Sport Rehabil. 2014, 23, 286-295. [CrossRef]

11. Massuça, L.M.; Fragoso, I.; Teles, J. Attributes of top elite team-handball players. J. Strength Cond. Res. 2014, 28, 178-186. [CrossRef] [PubMed] 
12. Marques, M.C.; van den Tilaar, R.; Vescovi, J.D.; Gonzalez-Badillo, J.J. Relationship between throwing velocity, muscle power, and bar velocity during bench press in elite handball players. Int. J. Sports Physiol. Perform. 2007, 2, 414-422. [CrossRef] [PubMed]

13. Ignjatovic, A.M.; Markovic, Z.M.; Radovanovic, D.S. Effects of 12-week medicine ball training on muscle strength and power in young female handball players. J. Strength Cond. Res. 2012, 26, 2166-2173. [CrossRef] [PubMed]

14. Marques, M.C.; Gonzalez-Badillo, J.J. In-season resistance training and detraining in professional team handball players. J. Strength Cond. Res. 2006, 20, 563-571. [PubMed]

15. Gorostiaga, E.M.; Izquierdo, M.; Iturralde, P.; Ruesta, M.; Ibáñez, J. Effects of heavy resistance training on maximal and explosive force production, endurance and serum hormones in adolescent handball players. Eur. J. Appl. Physiol. Occup. Physiol. 1999, 80, 485-493. [CrossRef] [PubMed]

16. Moher, D.; Liberati, A.; Tetzlaff, J.; Altman, D.G. PRISMA Group. Preferred reporting items for systematic reviews and meta-analyses: The PRISMA Statement. Open Med. 2009, 3, e123-e130.

17. Gorostiaga, E.M.; Granados, C.; Ibáñez, J.; Izquierdo, M. Differences in physical fitness and throwing velocity among elite and amateur male handball players. Int. J. Sports Med. 2005, 26, 225-232. [CrossRef]

18. Izquierdo, M.; Häkkinen, K.; Gonzalez-Badillo, J.J.; Ibáñez, J.; Gorostiaga, E.M. Effects of long-term training specificity on maximal strength and power of the upper and lower extremities in athletes from different sports. Eur. J. Appl. Physiol. 2002, 87, 264-271. [CrossRef]

19. Toumi, H.; Best, T.M.; Martin, A.; Poumarat, G. Muscle plasticity after weight and combined (weight + jump) training. Med. Sci. Sports Exerc. 2004, 36, 1580-1588. [CrossRef]

20. van Muijen, A.E.; Joris, H.; Kemper, H.C.G.; van Ingen Schenau, G.J. Throwing practice with different ball weights: Effects on throwing velocity and muscle strength in female handball players. J. Sports Med. Train. Rehabil. 1991, 2, 103-113. [CrossRef]

21. Ettema, G.; Glosen, T.; van den Tillaar, R. Effect of specific resistance training on overarm throwing performance. Int. J. Sports Physiol. Perform. 2008, 3, 164-175. [CrossRef]

22. Oxyzoglou, N.; Kanioglou, A.; Rizos, S.; Mavridis, G.; Kabitsis, C. Muscular strength and jumping performance after handball training versus physical education program for pre-adolescent children. Percept. Mot. Skills 2007, 104, 1282-1288. [CrossRef]

23. Holm, I.; Fosdahl, M.A.; Friis, A.; Risberg, M.A.; Myklebust, G.; Steen, H. Effect of neuromuscular training on proprioception, balance, muscle strength, and lower limb function in female team handball players. Clin. J. Sport Med. 2004, 14, 88-94. [CrossRef]

24. Carvalho, A.; Mourão, P.; Abade, E. Effects of Strength Training Combined with Specific Plyometric exercises on body composition, vertical jump height and lower limb strength development in elite male handball players: A case study. J. Hum. Kinet. 2014, 41, 125-132. [CrossRef]

25. Hermassi, S.; van den Tillaar, R.; Khlifa, R.; Chelly, M.S.; Chamari, K. Comparison of In-Season-Specific Resistance vs. A Regular Throwing Training Program on Throwing Velocity, Anthropometry, and Power Performance in Elite Handball Players. J. Strength Cond. Res. 2015, 29, 2105-2114. [CrossRef]

26. Granados, C.; Izquierdo, M.; Ibañez, J.; Bonnabau, H.; Gorostiaga, E.M. Differences in physical fitness and throwing velocity among elite and amateur female handball players. Int. J. Sports Med. 2007, 28, 860-867. [CrossRef]

27. Granados, C.; Izquierdo, M.; Ibáñez, J.; Ruesta, M.; Gorostiaga, E.M. Effects of an entire season on physical fitness in elite female handball players. Med. Sci. Sports Exerc. 2008, 40, 351-361. [CrossRef]

28. Sabido, R.; Hernández-Davó, J.L.; Botella, J.; Navarro, A.; Tous-Fajardo, J. Effects of adding a weekly eccentric-overload training session on strength and athletic performance in team-handball players. Eur. J. Sport Sci. 2017, 17, 530-538. [CrossRef]

29. van den Tillaar, R. Effect of different training programs on the velocity of overarm throwing: A brief review. J. Strength Cond. Res. 2004, 18, 388-396. [CrossRef]

30. Hermassi, S.; Wollny, R.; Schwesig, R.; Shephard, R.J.; Chelly, M.S. Effects of in-season circuit training on physical abilities in male handball players. J. Strength Cond. Res. 2019, 33, 944-957. [CrossRef]

(C) 2020 by the authors. Licensee MDPI, Basel, Switzerland. This article is an open access article distributed under the terms and conditions of the Creative Commons Attribution (CC BY) license (http://creativecommons.org/licenses/by/4.0/). 